\title{
Type I collagenases in bronchoalveolar lavage fluid from preterm babies at risk of developing chronic lung disease
}

\author{
D G Sweet, K J McMahon, A E Curley, C M O’Connor, H L Halliday
}

\begin{abstract}
Objective-To assess whether increased collagenolysis precedes severe chronic lung disease (CLD).

Methods-Matrix metalloproteinase-1 (MMP-1) and MMP-8 (enzymes that degrade type I collagen, the main structural protein of lung extracellular matrix) were measured by enzyme linked immunosorbent assay in 100 bronchoalveolar lavage samples taken during the first 6 postnatal days from 45 ventilated preterm babies < 33 weeks gestation. The median value for each baby was calculated. CLD was defined as an oxygen requirement after the 36th week after conception.

Results-MMP-8 levels in bronchoalveolar lavage fluid were higher (median $13 \mathrm{ng} / \mathrm{ml}$ ) in 20 babies who developed CLD than in 25 without CLD (median $2 \mathrm{ng} / \mathrm{ml}$ ). No MMP-1 was detected in any sample. Conclusions-MMP-8 can be detected in bronchoalveolar lavage fluid from preterm babies, and higher levels are found in those who later develop CLD. MMP-8 may contribute to lung injury that occurs as a prelude to CLD.

(Arch Dis Child Fetal Neonatal Ed 2001;84:F168-F171)
\end{abstract}

Keywords: chronic lung disease; lungs; collagen; extracellular matrix; matrix metalloproteinase; preterm

Improved antenatal care, along with advances in neonatal intensive care, have resulted in greater numbers of very preterm babies surviving who are at risk of developing chronic lung disease (CLD). ${ }^{1}$ The exact mechanisms underlying the lung injury and airways remodelling in CLD are not clear, but mechanical ventilation, oxygen toxicity, and inflammation all play a role. ${ }^{2-6}$ It is hypothesised that inflammation may directly damage the lung by increasing the release of proteolytic enzymes that degrade components of the lung interstitium. Both elastase $^{7}$ and matrix metalloproteinase (MMP)-9, which is a type IV collagenase, are increased in the lungs of babies who later develop CLD. ${ }^{8}$

Lung biomechanical properties are largely dependent on the correct distribution and abundance of the extracellular matrix which is also crucial in maintaining the alignment and differentiated state of the various epithelial cell layers lining the airways. ${ }^{9}$ Preterm babies born before 28 weeks gestation are just starting to develop a pulmonary capillary bed and are beginning the process of alveolarisation of the distal saccules. Lung injury during this crucial period of remodelling may result in arrest of lung development. ${ }^{10}$

MMPs are enzymes required to support and maintain the extracellular matrix. ${ }^{112}$ Type I collagen is the major structural protein of the extracellular matrix, and MMP-1 and MMP-8 are the only proteinases that degrade type I collagen. ${ }^{13}$ Excess MMP-8 activity has been implicated in adult lung diseases such as emphysema ${ }^{14}{ }^{15}$ and bronchiectasis. ${ }^{16}$ In this study, we measured MMP-1 and MMP-8 levels in bronchoalveolar lavage (BAL) fluid from preterm babies within the first week of life to determine if excessive type I collagenolysis is implicated in the development of severe CLD.

Patients and methods

This was a prospective study of babies born in the Royal Maternity Hospital in Belfast. The research ethics committee of The Queen's University of Belfast gave approval for the study, and parental consent was obtained before babies were enrolled.

Forty five babies of less than 33 weeks gestation were recruited when they required intubation and mechanical ventilation in the first 6 days of life. Gestation was estimated by duration of amenorrhoea combined with early ultrasound measurement. Serial BAL was performed daily whenever possible from the time of intubation until extubation or the 6th day of life, whichever was soonest. Babies were only studied when clinically stable and not during the 12 hours after a dose of surfactant. After extubation, the babies' progress was monitored until discharge or the 36th week after conception. For the purposes of this study, severe CLD was defined as a requirement for supplemental oxygen to maintain oxygen saturation measured by pulse oximetry above $92 \%$ when at rest after the 36th week after conception. The severity of the initial respiratory disease was compared using the median arterialalveolar oxygen tension ratio (a/A ratio) in the first 24 hours of life. This was calculated from arterial blood gases using the equation:

$$
\text { a } / \text { A ratio }=\mathrm{PaO}_{2} /\left(\left(96 \times \mathrm{FIO}_{2}\right)-\mathrm{PaCO}_{2}\right)
$$

where $\mathrm{PaO}_{2}$ and $\mathrm{PaCO}_{2}$ are expressed in $\mathrm{kPa}$ and $\mathrm{FIO}_{2}$ as a fraction of 1 .

METHOD FOR BAL

BAL was performed in a standardised way using a well established technique, ${ }^{5}$ although unlike the study by Kotecha et al no marker of dilution was used. This is in accordance with the most recent European Respiratory Society guidelines on BAL. ${ }^{17}$ In summary, $1 \mathrm{ml} / \mathrm{kg}$ sterile $0.9 \%$ saline was instilled using a $2 \mathrm{ml}$ 
Table 1 Clinical characteristics of babies studied

\begin{tabular}{llll}
\hline & $C L D(n=20)$ & No CLD (n=25) & p Value \\
\hline Gestation (weeks) & $25.2(1.5)$ & $28.4(2.5)$ & $<0.0001^{\star}$ \\
Birth weight (g) & $759(189)$ & $1312(416)$ & $<0.0001^{\star}$ \\
Median a/A ratio first 24 hours & $0.37(0.11)$ & $0.40(0.18)$ & $0.79^{\star}$ \\
Sex (male : female) & $12: 8$ & $15: 10$ & $0.72 \dagger$ \\
\hline
\end{tabular}

Results are shown as mean (SD).

*Mann-Whitney U test.

†Fishers exact test.

CLD, Chronic lung disease.

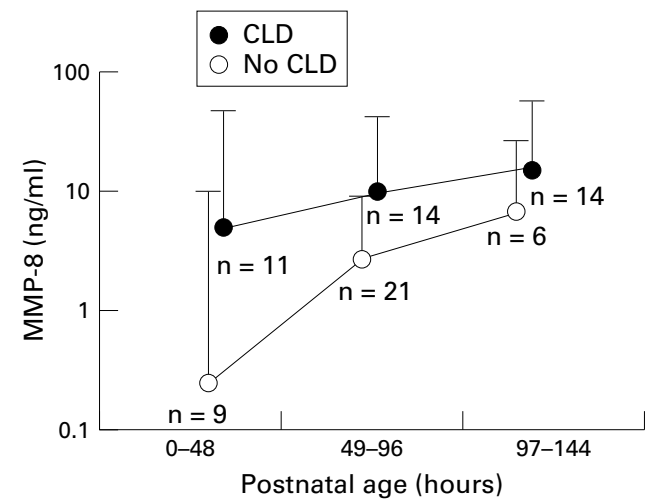

saline was instilled and immediately aspirated back into the syringe. The median volume of saline returned was $0.7 \mathrm{ml}$ (interquartile range (IQR) $0.5-1.2 \mathrm{ml}$ ). The sample was clarified by centrifugation at $1500 \mathrm{~g}$ for five minutes at room temperature, and the supernatant immediately frozen at $-70^{\circ} \mathrm{C}$ and kept for subsequent analysis.

LABORATORY METHODS

Samples were assayed for MMP-1 and MMP-8 by commercial enzyme linked immunosorbent assay (ELISA) in accordance with the manufacturer's instructions (Amersham International, Amersham, Bucks, UK). The ELISA systems measure total levels of their respective antigens, including latent and active forms of the MMPs and $\mathrm{MMP} /$ tissue inhibitor of metalloproteinase (TIMP) complexes. The sensitivity of the MMP-8 assay was 0.032 $\mathrm{ng} / \mathrm{ml}$ (range $0.032-4 \mathrm{ng} / \mathrm{ml}$ ), and that of the MMP-1 assay was $1.7 \mathrm{ng} / \mathrm{ml}$ (range $1.7-100$ $\mathrm{ng} / \mathrm{ml}$ ). The ability of the MMP-1 assay to detect this enzyme in dilute biological fluids was confirmed by analysis of supernatants from phorbol myristrate acetate stimulated alveolar macrophages. All samples were assayed in duplicate.

STATISTICAL ANALYSIS

To simplify statistical analysis of changes over time, postnatal age was divided into 48 hour time periods. If more than one sample from an individual baby was taken within a time period, the median result was used. Median MMP levels were calculated for each baby and used as the summary statistic. MMP-8 levels were not normally distributed, so non-parametric statistical tests were used. The logarithm of MMP-8 concentration was normally distributed and used in the logistic regression analyses. Spearman correlations, Mann-Whitney U tests, Wilcoxon signed rank test, $\chi^{2}$ and regression analysis were all calculated using STATVIEW for Windows version 4.571996 (Abacus Concepts Inc, Berkeley, California, USA).

\section{Results}

A total of 100 BAL samples were collected for analysis. Twenty of the 45 babies studied developed severe CLD. They were less mature and lighter at birth than those who did not, but there was no significant difference in initial severity of respiratory distress, as assessed by the a/A $\mathrm{PO}_{2}$ ratio during the first 24 hours (table 1). There was a preponderance of boys in both groups.
Figure 1 Postnatal changes in matrix metalloproteinase-8 (MMP-8) concentrations in bronchoalveolar lavage (BAL) fluid from babies who later developed chronic lung disease (CLD) and those who did not. Data depicting medians and 75th percentiles are presented on a log scale. $M M P-8$ levels were higher in babies who later developed $C L D$. No CLD v CLD: at 0-48 hours, median 0.25 v 5 $\mathrm{ng} / \mathrm{ml}(p=0.16)$; at 49-96 hours, median 3 v $9 \mathrm{ng} / \mathrm{ml}$ $(p=0.006)$; at 97-144 hours, median 7 v $14 \mathrm{ng} / \mathrm{ml}$ $(p=0.51)$

MMP-1 was not detected in any BAL BAL samples. For all 100 BAL samples, MMP-8 levels did not change significantly with increasing postnatal age $(r=0.17 ; \mathrm{p}=0.1)$. Analysis of MMP-8 levels was also carried out on infants who had samples taken during both the $0-48$ and $49-96$ hour periods. MMP-8 levels did not vary significantly with postnatal age in this group (median (IQR) at 0-48 hours, 2.5 (0.03-17.2) ng/ml; at 49-96 hours, 8.1 (4.531.9) $\mathrm{ng} / \mathrm{ml}$ ). Each baby had on average two BAL samples taken (range 1-5). Babies who subsequently developed CLD tended to have more samples taken over the six day period. MMP-8 levels were higher throughout the first six days in babies who developed CLD, but because of the small number of samples, this result was only statistically significant between 49 and 96 hours of age (fig 1).

The use of median MMP-8 levels as the summary statistic for each baby showed that MMP-8 levels were negatively correlated with gestational age $(r=-0.35 ; \mathrm{p}=0.01)$ and birth weight $(r=-0.32 ; \mathrm{p}=0.02)$. Median MMP-8 levels were about 10-fold higher in babies who subsequently developed CLD (fig 2).

To avoid the bias of more samples being taken from babies who developed CLD, the 74 samples taken within the first four postnatal days were also analysed in isolation. Forty two babies were available for study, of whom 17 developed CLD. The proportion of babies in whom MMP-8 was detected was significantly higher in the CLD group than in the no CLD group (15/17 (88\%) v 14/25 (56\%); p = 0.026) and the difference in MMP-8 levels remained significantly higher (median (IQR) 9 (5-32) $v$ $2(0-6) \mathrm{ng} / \mathrm{ml} ; \mathrm{p}=0.0039)$.

All 45 babies were used in a multiple logistic regression model to assess factors that could influence the likelihood of developing CLD. Gestation, birth weight, median a/A ratio on day 1 , sex, and log MMP-8 were tested, and of these only gestation $(\mathrm{p}<0.0001)$, birth weight $(\mathrm{p}<0.0001)$, and $\log \mathrm{MMP}-8(\mathrm{p}=0.0012)$ were individually predictive of CLD. samples. MMP-8 was detected in 68 of the 100 


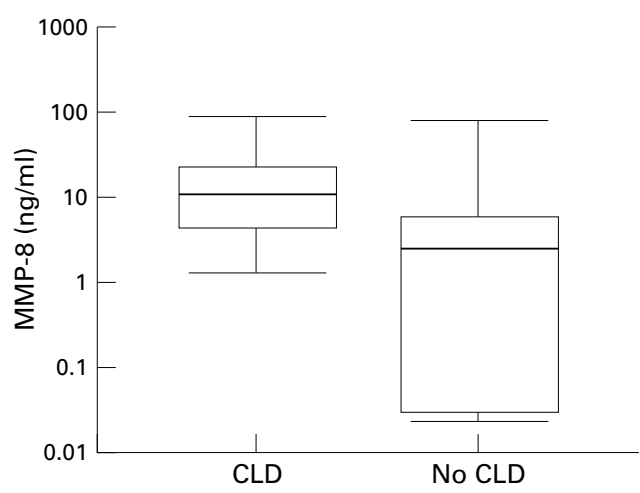

Figure 2 By using all the bronchoalveolar lavage samples, median matrix metalloproteinase-8 (MMP-8) concentration was calculated for each baby. The distribution of these medians is indicated using box and whisker plots that show medians, 25th and 75th percentiles, and the full range. Median MMP-8 levels were significantly higher in babies who subsequently developed severe chronic lung disease (CLD) than in those who did not (13 v $2 \mathrm{ng} / \mathrm{ml}$; $p=0.001)$.

\section{Discussion}

Inflammation and neutrophil chemotaxis are essential prerequisites for the development of CLD. Neutrophil counts in tracheal aspirate are 20-fold higher on the third day of life in babies who later develop CLD than in those who do not. ${ }^{18}$ Neutrophil influx into the lungs probably contributes to tissue degradation by release of proteinases. We have previously shown that type IV collagenase (MMP-9) levels are increased in BAL fluid from babies who subsequently develop CLD and that there is an imbalance between MMP-9 and one of its major inhibitors, TIMP-1. ${ }^{8}$ Type IV collagen is the major constituent of basement membranes. Disruption of the basement membranes may explain the increased lung permeability observed in babies who develop $\mathrm{CLD},{ }^{4}$ but cannot explain all of the gross structural changes found in the lungs of babies with established CLD. We hypothesised that early destruction of the interstitial collagens in neonatal respiratory distress syndrome and matrix destruction may also be an important precursor to the development of structural remodelling of the airways. ${ }^{19}$

MMP-1 is a $55 \mathrm{kDa}$ enzyme also known as interstitial collagenase or fibroblast type collagenase. It is released by a wide variety of stromal cells including fibroblasts, endothelial cells, osteoblasts, and chondroblasts. It will digest all major components of the extracellular matrix but it will preferentially degrade type III collagen. Its main function is during tissue homoeostasis and wound repair. MMP-8, or neutrophil collagenase, is a $75 \mathrm{kDa}$ enzyme which is predominantly expressed by neutrophils, although some studies suggest that it can be expressed by other cells. ${ }^{20}{ }^{21} \mathrm{MMP}-8$ also digests all major components of the extracellular matrix, but will preferentially degrade type I collagen. ${ }^{22}$ These two collagenases have a unique ability to cleave the native helix of fibrillar collagen by hydrolysing a single bond located one quarter length from the collagen $\mathrm{C}$ terminus. ${ }^{23}$ This process yields one quarter and three quarter fragments that at body temperature will spontaneously denature into randomly coiled gelatin peptides. Other members of the MMP family will then further degrade these into hydroxyproline. We have shown a 10 -fold increase in median MMP-8 concentrations in BAL fluid from babies who subsequently develop severe CLD, suggesting that this enzyme may contribute to the early tissue destruction. Dissolution of the main structural framework of the lung matrix is likely to result in disorganisation of the lung architecture during healing. This may explain why lungs of babies with CLD have fewer than normal larger alveoli. ${ }^{24}$ We did not detect MMP-1 in any of the neonatal samples. This may reflect the less sensitive nature of the assay system (1.7 $\mathrm{ng} / \mathrm{ml}$ for MMP-1 $v 0.032 \mathrm{ng} / \mathrm{ml}$ for MMP-8). Although MMP-1 levels within the assay range were measurable in conditioned medium from phorbol myristrate acetate stimulated macrophages, even these were at the lower end of the assay standard curve $(<10 \mathrm{ng} / \mathrm{ml})$. Given the dilution effect inherent in sampling airway fluids by BAL, it is likely that the failure to detect MMP-1 was a consequence of this dilution. Although sample availability constraints did not allow for assessment of lining fluid dilution, such measurements would not have assisted in addressing this issue.

As indicated above, the major source of MMP-8 is the inflammatory neutrophil. Neutrophil derived MMPs are stored within intracellular granules and are released during neutrophil activation along with other neutrophil products, including the reactive oxygen radicals of the oxidative burst. ${ }^{25} \mathrm{MMP}$ activity may also be influenced by oxidative stress, which is believed to be important in the pathogenesis of CLD. In a rat model, hyperoxia has been shown to increase expression of mRNA for MMP-8, with associated increased collagenase activity. ${ }^{26}$ Oxygen free radicals can activate pro-MMP-3, a proteinase that in turn will activate the latent form of MMP-8 in the tissues. ${ }^{27}$ TIMP-1, a major inhibitor of MMP activity, can be inactivated by oxygen free radicals, which may further tip the balance in favour of matrix degradation. ${ }^{28}$

A predictive test for CLD would be useful for determining which babies may benefit from early interventions to limit inflammation. In this study, MMP- 8 was detected in $88 \%$ of the babies who developed CLD compared with $56 \%$ of those who did not develop CLD, but it is clear that, although this difference is statistically significant, it is unlikely that this measure in isolation will prove to be a useful predictive test.

In summary, we detected MMP-8, but not MMP-1, in BAL fluid from preterm babies at risk of developing CLD. There was a greater likelihood of MMP-8 being present if the baby subsequently developed severe CLD, and median MMP-8 levels were about 10-fold higher in the babies who developed CLD. We speculate that MMP-8 activity in the preterm lung may contribute to the lung damage that occurs in the early stages of CLD. 
This project was supported by a grant from the Northern Ireland Mother \& Baby Appeal.

1 Parker RA, Lindstrom DP, Cotton RB. Improved survival accounts for most but not all of the increase in bronchopaccounts for most but not all of the increase in

2 Philip AGS. Oxygen plus pressure plus time: the etiology of bronchopulmonary dysplasia. Pediatrics 1975;55:44-50.

3 Saugstad OD. Chronic lung disease: the role of oxidative stress. Biol Neonate 1998;74(suppl 1):21-8.

4 Groneck P, Gotze-Speer B, Oppermann M, et al. Association of pulmonary inflammation and increased microvascular permeability during the development of bronchopulmonary dysplasia: a sequential analysis of
inflammatory mediators in respiratory fluids of high risk preterm neonates. Pediatrics 1994;93:712-18.

5 Kotecha S, Chan B, Azam N, et al. Increase in interleukin-8 and soluble intercellular adhesion molecule-1 in bronchoalveolar lavage fluid from premature infants who develop chronic lung disease. Arch Dis Child Fetal Neonatal Ed 1995;72:F90-6.

6 Brus F, van Oeveren W, Okken A, et al. Activation of circulating polymorphonuclear leukocytes in preterm infants lating polymorphonuclear leukocytes in preterm infants with severe idiopathic res
atr Res 1996;39:456-63.

7 Watterberg KL, Carmichael DF, Gerdes JS, et al. Secretory leukocyte protease inhibitor and lung inflammation in
developing bronchopulmonary dysplasia. $f$ Pediatr developing bron

8 Sweet DG, Pizzoti J, Wilbourn M, et al. Matrix metalloproteinase-9 (MMP-9) in the airways of infants at risk of developing chronic lung disease (CLD). Eur Respir f 1999;14(suppl 30):248s.

9 Rannels DE, Rannels SR. Influence of the extracellular matrix on type 2 cell differentiation. Chest 1989;96:16573.

10 Jobe AJ. The new BPD: an arrest of lung development. Pediatr Res 1999;46:641-3.

11 Dean DD, Martel-Pelletier J, Pelletier JP, et al. Evidence of metalloproteinase and metalloproteinase inhibitor imbalance in human osteoarthritic cartilage. Clin Invest 1989;84:678-85.

12 O'Connor CM, FitzGerald MX. Matrix metalloproteases and lung disease. Thorax 1994;49:602-9.

13 Davidson JM. Biochemistry and turnover of lung interstitium. Eur Respir f 1990;3:1048-68.

14 Finlay GA, O'Driscoll LR, Russell KJ, et al. Matrix metalloproteinase expression and production by alveolar macrophages in emphysema. Am $\mathcal{F}$ Respir Crit Care Med 1997;156:240-7

15 Betsuyaku T, Nishimura M, Takeyabu K, et al. Neutrophil granule proteins in bronchoalveolar lavage fluid from subjects with subclinical emphysema. Am f Respir Crit Care Med 1999;159:1985-91.
16 Sepper R, Kontinnen YT, Ding Y, et al. Human neutrophil collagenase (MMP-8), identified in bronchiectasis BAL fluid, correlates with severity of disease. Chest 1995;107:1641-7

17 De Blic J, Midulla F, Barbato A, et al. Bronchoalveolar lavage in children. ERS Task Force on bronchoalveolar lavage in children. European Respiratory Society. Eur Respir 7 2000;15:217-31.

18 Merritt TA, Stuard ID, Puccia J, et al. Newborn tracheal aspirate cytology: classification during respiratory distress syndrome and bronchopulmonary dysplasia. 7 Pediatr 1981;98:949-56.

19 Murch SH, Costeloe K, Klein NJ, et al. Mucosal tumor necrosis factor alpha production and extensive disruption of sulfated glycosaminoglycans begin within hours of birth in neonatal respiratory distress syndrome. Pediatr Res 1996;40:484-9.

20 Cole AA, Chubinskaya S, Schumacher B, et al. Chondrocyte matrix metalloproteinase-8. Human articular chondrocytes express neutrophil collagenase. 7 Biol Chem cytes express neutr.

21 Hanemaaijer R, Sorsa T, Konttinen YT, et al. Matrix metalloproteinase- 8 is expressed in rheumatoid synovial fibroblasts and endothelial cells. Regulation by tumor necrosis factor-alpha and doxycycline. 7 Biol Chem 1997;272:31504-9.

22 Mallya SK, Mookhtiar KA, Gao Y, et al. Characterisation of 58-kilodalton human neutrophil collagenase: comparison with human fibroblast collagenase. Biochemistry 1990;29:10628-34

23 Fields GB, van Wart HE, Birkedal-Hansen H. Sequence speificity of human skin fibroblast collagenase. Evidence for the role of collagen structure in determining the collagenase cleavage site. F Biol Chem 1987;262:6221-6.

24 Hislop AA, Wigglesworth JS, Desai R. Alveolar development in the human fetus and infant. Early Hum Dev 1986;13:1-11.

25 Hibbs MB, Hasty KA, Kang AH, et al. Secretion of collagenolytic enzymes by human polymorphonuclear leukocytes. Coll Relat Res 1984;4:467-77.

26 Devaskar UP, Taylor W, Govindrajan R, et al. Hyperoxia induces interstitial (type I) and increases type IV collagenase mRNA expression and increases type I and IV collagenolytic activity in newborn rat lung. Biol Neonate 1994;66:76-85.

27 Nagase H. Activation mechanisms of matrix metalloproteinases. Biol Chem 1997; 378: 151-160.

28 Frears ER, Zhang Z, Blake DR, et al. Inactivation of tissue inhibitor of metalloproteinase-1 by peroxynitrite. FEBS Lett 1996;381:21-4. 